\title{
Research on Mechanical Problems of Rotation Body Motion of Ball Mills
}

\author{
Zhiqiang Xu \\ Department of Physics and Technology, Kunming University, Kunming, 650214, China \\ 2793436659@qq.com
}

Keywords: ball mill; rotation body; dropping motion; crushing medium; steel ball;

\begin{abstract}
When ANSYS finite element analysis of the ball mill rotation body is conducted, the force of the ball mill rotation body should be analyzed first. The force of the rotation body is closely related to the grinding action of the ball mill. The grinding effect of the ball mill is completed based on the movement of the medium in its rotation body. Therefore, it is necessary to study the motion of the medium in the rotation body in order to study the grinding effect of the ball mill. On the basis of it, the force of the rotation body will be studied. This paper studies the basic problems of mechanics.
\end{abstract}

\section{Introduction}

The material pulverization of the ball mill is achieved by impacting, grinding and stripping the material through the movement of the crushing medium. The motion pattern and motion law of the crushed medium are the basis for studying the productivity, optimizing the parameters and improving the milling efficiency of the ball mill. Therefore, the primary task of studying the grinding theory of ball mill [1-3] is to study the motion pattern of grinding medium and pattern of motion. The movement of the medium in the rotation body of the ball mill is a complicated process. Its movement depends on the speed of the mill cylinder, medium filling rate, mill liner, ball-to-batch ratio, slurry concentration, etc. [5] [6], especially the rotation speed of the ball mill and the filling rate of the medium [4] .The primary task is to analyze the movement law of the crushing medium in the rotation body of the ball mill to select the working parameters economically and to improve the working efficiency of the mill. As far as the motion state of the medium in the ball mill is concerned, a lot of experimental researches have been carried out and many different viewpoints have been put forward. The three typical motion states of sluice type, dropping type and centrifugal type are determined from several motion states of the ball mill crushing medium. The grinding effect- one of the three typical motion forms is analyzed [7].

\section{Dropping Motion State}

When the crushing medium moves in the high-speed rotation body, the motion trajectory at any layer of the medium can be divided into two segments, as shown in Figure 1. When rising, the medium is circumvented from the dropping point $A_{1}$ to the detachment point $A_{5}$, which shows the circular trajectory movement. However, from the detachment point $A_{5}$ to the dropping point $A_{1}$, it falls on a parabolic trajectory between point $A_{5}$ and point $A_{1}$, followed by circular trajectory movement, repeatedly cycles. Under the friction between the inner wall of the cylinder (liner) and the outermost medium, the medium on the external layer moves along a circular path, creating friction among adjacent layers of media. Therefore, the media of the inner layers also move along a circular trajectory of a concentric circle, which rotate together with the cylinder as a whole. The friction depends on the coefficient of friction and the positive pressure acting on the inner wall of the cylinder (or the adjacent medium layer). The positive pressure is generated by the radial component of force $\mathrm{N}$ and Centrifugal force $C$. The tangential component of gravity $T$ has a tendency to rotate the medium in the direction opposite to the direction of rotation of the cylinder. If the friction moment to the cylinder is greater than that of the tangential component $T$ to the center of the cylinder, there is no relative sliding between the medium and the wall of the cylinder or the 
medium layer. On the contrary, there is relative sliding.

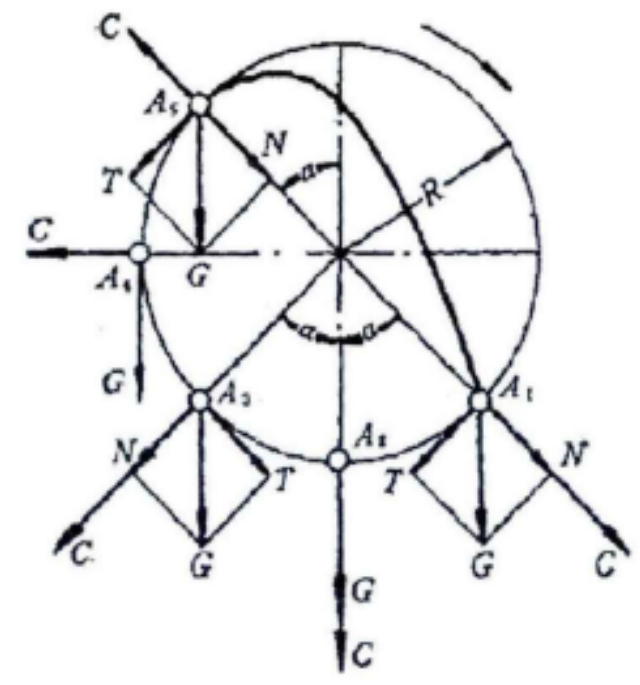

Figure 1. The motion trajectory of the medium in the dropping state

The coefficient of friction depends on the nature of the ore, the shape of the inner surface of the cylinder and the concentration of the slurry. When the friction coefficient is constant, there is not much crushing medium in the cylinder, the rotating speed of the rotating body is low, and the friction is small to a certain extent due to the small positive pressure, the medium will slide relatively along the cylinder wall. There is also relative sliding between the medium layers. Meanwhile the medium also rotates around its own geometric axis. In any layer of the media, the movement of each medium along a circular path is not simply caused by the friction acting on this medium alone but relies on the friction of the entire media. This medium is driven acting only as a group of all the rotating media, which is supported" by the media of the same layer behind. When in a dropping state, materials are crushed both by the impact force generated under the medium community and by partial grinding. The ball mill works in this kind of state.

\section{The Motion Trajectory of the Crushing Medium in the Dropping State}

When the ball mill is working, the material in the cylinder is crushed mainly by impact and honing of the dropping motion of the medium. It can be seen that the dropping motion of the crushing medium is a better method of grinding. Therefore, the motion of the crushing medium in the dropping state will be systematically analyzed [7-9]. When the ball mill starts working, the ball and the cylinder rotate together due to the centrifugal force and the friction. The motion trajectory of the ball on any layer is centered on the center of the cylinder, with $\mathrm{R}$ being the radius of the radius (the radius of the rotating layer where the ball is located). When the ball rotates together with the cylinder and is lifted to a certain height, the centrifugal force of the ball is less than the centripetal force of the ball weight, the ball moves away from the wall of the cylinder at a preliminary velocity $V$ (circumferential speed of the cylinder) for parabolic motion. After falling, the ball returns to the circular trajectory. During this process, the ball moves back and forth in the ball mill in a circular and parabolic trajectory. Studying the motion law of the steel ball in the ball mill, the motion of a steel ball in the outermost layer of the cylinder should be analyzed. The following assumptions are made to simplify the discussion:

(1) The movement of the steel balls is completely similar in each vertical section of the axial direction of the cylinder, and the section is independent;

(2) The steel ball remains relatively static to the inner wall of the cylinder, and so does the steel ball to the other steel ball, without relative sliding phenomenon;

(3) Since the diameter of the steel ball is much smaller than the inner diameter of the cylinder, and the diameter of the steel ball is omitted, the radius of gyration of the outer ball can be expressed by the inner radius of the cylinder. On the basis of the hypothesis, the motion theory of the crushing 
medium of the ball mill in the dropping state is studied.

\section{The Trajectory Equation of the Steel Ball during the Dropping Motion}

When the steel ball is in a parabolic motion, its motion is divided into two steps as shown in Figure 2. The circular motion around the circular track $B A$ from point $A$ to point $B$. In the figure, point $A$ is called the detachment point, and point $B$ is called the dropping point. $\alpha$ is the angle between the line of centre $O A$ and the vertical axis, indicating the detachment angle at which the ball detaches from the original trajectory. $\beta$ is the angle between the line of centre $O B$ and the horizontal axis, which is called the dropping angle. As shown in Figure 2, the radius of the cylinder (the unit is $\mathrm{m}$ ) is represented by $R$. When the ball in a circular motion reaches point $A$, the centrifugal force $C$ of the ball is equal to the radial component $N$ of the ball weight $G$. Its tangential component force $T$ is offset by the thrust of a subsequent balls. If the ball crosses point $A$, the ball falls off the cylinder along the parabolic trajectory at tangential speed $v$.

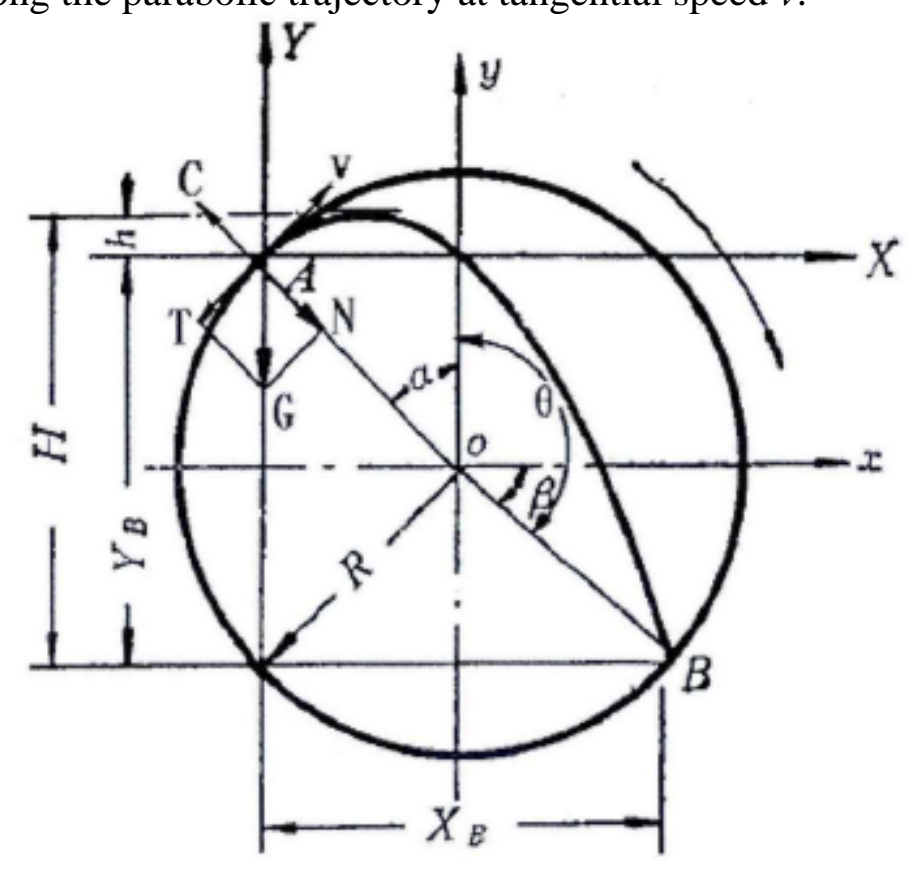

Figure 2. The trajectory and force status of the ball

Take point $A$ as the coordinate origin of the Cartesian coordinate system $X A Y$. In this coordinate system, the circle must be at the center Point $O$ of the cylinder in the ball mill, the curve equation with radius $R$ is:

$$
(X-R \sin \alpha)^{2}+(Y+R \cos \alpha)^{2}=R^{2}
$$

Equation (1) is the equation of motion of the ball in a circular motion in the XAY coordinate system. If $\alpha$ indicates the angle at which the steel ball begins to make a dropping motion, that is, the angle at which the circular motion is removed (the angle of detachment), the balanced relationship at the detachment point is:

$$
\begin{gathered}
C=G \cos \alpha \\
C=m R \omega^{2}=m \frac{V^{2}}{R}
\end{gathered}
$$

Substitute the $C$ value into the above formula, and get:

$$
\frac{V^{2}}{R}=g \cos \alpha
$$


Where: $m$ - the quality of the ball;

$v$-the speed of the ball, $v=\frac{\pi R n}{30}, \mathrm{~m} / \mathrm{s}$;

$n$-the rotation speed of the cylinder, $\mathrm{r} / \mathrm{min}$;

$g$-gravity acceleration, $\mathrm{m} / \mathrm{s}^{2}$.

After the steel ball is detached from point A, according to the law of parabolic motion, its horizontal distance $\mathrm{x}$ and vertical distance $\mathrm{y}$ are:

$$
\begin{gathered}
X=(v \cos \alpha) t \\
Y=(v \sin \alpha) t+\frac{1}{2} g t^{2}
\end{gathered}
$$

Eliminate the value of $\mathrm{t}$ in the above equation and get:

$$
Y=X \tan \alpha-\frac{g X^{2}}{2 v^{2} \cos ^{2} \alpha}
$$

Substitute formula (3) into the above formula, the equation for the steel ball to make the dropping motion in the coordinate XAY is obtained.

$$
Y=X \tan \alpha-\frac{X^{2}}{2 R \cos ^{3} \alpha}
$$

\section{Conclusion}

This paper analyzes not only one of the three typical motion patterns of the medium in the cylinder of the ball mill, but also the movement of the internal crushing medium in the ball mill under the falling motion mode. The trajectory equation of the crushing medium for the dropping motion is obtained. The basic force of the cylinder is also analyzed in detail, which lays the foundation for finite element analysis.

\section{References}

[1] Belov, Brand. Grinding [M]. Translated by Nailiang Dong, Entang Hu, etc., Qianning: Suining People's Publishing House, 1954

[2] Crushing and Grinding Machinery Writing Group. Grinding Machinery [M], Beijing: Mechanical Industry Press, 1978.

[3] Jiawen Yang. Crushed Mining and Grinding Technology [M]. Metallurgical Industry Press, 2006: 50-70.

[4] Lam Eck N. S, Kiangi K. Effects of Grinding Media Shapes on Load Behavior and Mill Power in a Dry Ball Minerals Engineering, 2006,(9):135-1361.

[5] Xiulan Ouyang, Xiaofang Hu, Guosheng Gai. In the Actual Process, the Grinding State of the Grinding Media of the Ball Mill is studied [J]. Metal Mine Mountain, 2004: 293-295.

[6] Xinmin Tang, Mingsheng Liu, Duoyi Wu. Relationship between the Ball mill Filling Rate and Grinding Capacity [J]. Mining Machinery, 2002, 4: 18-21.

[7] Enpu Zhou Mining machinery (part of Mineral Processing Machinery) [M], Beijing: Metallurgical Industry Press, 1979.

[8] P. P. Chattopadhyay, I. Manna, S. Talapatra, S. K. Pabi. A Mathematical Analysis of milling Mechanics in Aplanetary Ball Mill [J]. Materials Chemistry and Physics, 2001(68):85-94.

[9] M. Abdellaoui, E. Gaffet. The Physics of Mechanical Alloying in a Planetary Ball Mill Mathematical Treatment [J]. Acta Metal Master. Vol. 43, No:3, 1995:1087-1098. 